\title{
Ein irrationales Behandlungskonstrukt
}

Max Geiser

Christian Larsen, Leiter des Spiraldynamik Med Center Zürich, nimmt auf Seite 1973 Stellung.

Korrespondenz: Prof. Dr. med. Max Geiser Gossetstrasse 49 CH-3084 Wabern
Als Kritiker von angeblich heilerischen alternativen Praktiken habe ich mich um Informationen über die sogenannte Spiraldynamik bemüht. Von ihren Erfindern wird sie als innovative Physiotherapieform zur Behandlung von Krankheiten und Beschwerden im Bereiche des Bewegungsapparates angepriesen und in mehrtätigen Kursen vermittelt. Ich informierte mich über die Spiraldynamik mit Hilfe eines in der Medical Tribune vom 13.3.2009 erschienenen Beitrags, der Fernsehsendung Medical Talk auf TeleBärn, durch das Internet mit den letzten Aktualisierungen vom 4.2.2011 und vom 15.5.2011, durch den Artikel «Trainieren statt Operieren?» [1] des medizinischen Leiters des Spiraldynamik Med Centers in Zürich (das auf «nicht operative Orthopädie und Physiopädagogik» spezialisiert ist), einen Leserbrief desselben Autors [2] und in den von der «Spiraldynamik intelligent movement» zur Verfügung gestellten Broschüren «Tageskurse» und «Akademie» (Aus- und Weiterbildungsprogramme). Aus diesen Informationsquellen ergibt sich Folgendes:

Der Mitbegründer der «Spiraldynamik» Dr. med. Christian Larsen hat in Basel Medizin studiert und sich zum Facharzt Allgemeinmedizin weitergebildet. Er begann vor 31 Jahren gemeinsam mit der französischen Physiotherapeutin Yolande Deswarte («coordination motrice», Paris) die Globalbewegungen des menschlichen Körpers zu erforschen, bis sie das «komplette Bewegungsmodell des «Bauplan Mensch〉 erarbeitet hatten». Es handelt sich um eine «Neuinterpretation der menschlichen Anatomie». Dr. Larsen nennt als sein Forschungs- und Spezialgebiet Anatomie und Biomechanik aus der Sicht der Evolutionsgeschichte und der «Nicht-operativen Orthopädie». Auf ausgedehnten Studienreisen in Alaska, Tibet, Japan, China, in der Sahara und anderen Ländern erforschte er die Heilmethoden anderer Kulturen. Dr. Larsen ist Leiter des Spiraldynamik Med Centers der Privatklinik Bethanien in Zürich, Vizepräsident des Verwaltungsrates der Spiraldynamik AG, internationaler Dozent, Bestsellerautor und Preisträger. Seine Fachbücher und Patientenratgeber stossen auch in Deutschland, Österreich und Japan auf breites Interesse. Die Spiraldynamik-Bewegung umfasst 3000 ausgebildete Fachkräfte in der Schweiz, im deutschsprachigen Ausland und in Tschechien. Aus dem Beitrag «Trainieren statt operieren?» [1] vernimmt der Leser, dass die nicht-operative Chirurgie gemäss dem Prinzip «trainieren statt operieren» seit 10 Jahren unter dem Namen «Spiraldynamik» umgesetzt wird. Gemäss einem Leserbrief [2] gelang es der Spiraldynamik Med Center AG, in Basel,
Bern und Zürich ein diagnostisch-therapeutisches Zentrum unter orthopädisch-fachärztlicher Leitung zu gründen. Dem Med Center in Zürich wurden im letzten Jahr von über 200 Ärzten (auch der Schulthessklinik und der Uniklinik Balgrist) Patienten zugewiesen.

Im Internet (am 4.2.2011 aktualisiert) wird der Leser mit verworren wirkenden Aussagen wie «Das Kreuz ist entscheidend für das Wohlergehen von Rücken und Bandscheiben», «Ein gesundes Hüftgelenk ist eine Frage von Knowhow», «Die Hand macht den Menschen zum Menschen» und «Das Knie ist Befehlsempfänger - kluger Gebrauch vorausgesetzt». Er vernimmt, dass die Spiraldynamik zur Beschreibung von Bewegungsabläufen die Helix (Spirale) und als dynamisches Prinzip die Welle benutzt, eine dreidimensionale Bewegung von Kopf bis Fuss ist und sich für Wirbelsäulen-, Schulter-, Hüft-, Knie- und Fussbeschwerden eignet, «überall und immer anwendbar» und zudem Name und Wortmarke eines Schweizer Franchise-Unternehmens für Physiotherapeuten ist. In den Broschüren «Tageskurse» und «Akademie» werden Kurse für Kiefer, Nacken, Schultern, Hände, Brustkorb, Kreuz, Becken, Hüften, Knie und Füsse angeboten. Dies für einen halben bis zu 30 Tagen für 100 bis 3000 Franken. Die Kurse vermitteln Physiotherapie, Ergotherapie, «dreidimensionales Training», Tanz, Yoga und «Körperarbeit».

\section{Beurteilung der «Spiraldynamik»}

Nach Kenntnisnahme der zitierten Informationen hat der Leser die Wahl, die Spiraldynamik entsprechend dem Erfinder als revolutionäres Konzept der Diagnostik und Therapie der im Bereich des Bewegungsapparates auftretenden Krankheiten, Deformitäten und Beschwerden zu bewerten. Oder er beurteilt die Spiraldynamik als Produkt eines Allgemeinmediziners ohne Facharzttitel FMH für Orthopädie, der die anerkannten anatomischen, physiologischen und pathologischen Fakten im Bereich des Bewegungsapparates nicht beachtet und durch ein Fantasiegebilde ersetzt und mit Kursen verbreitet.

Aus meiner Sicht hat ein nicht orthopädisch ausgebildeter Facharzt für Allgemeinmedizin zusammen mit einer Physiotherapeutin eine Therapiemethode mit der Bezeichnung «Spiraldynamik» ersonnen, die ein spiralförmiges Bewegungsmuster propagiert, das gegen alle im Bewegungsapparat vorkommenden Beschwerden wirken und der «Gesundheitsförderung» dienen soll. Die «Spiraldynamik» entspricht einer Allheilmethode wie viele andere alternative Prakti- 
ken. Die langen Behandlungszeiten über Monate hinweg und das Fehlen eines Wirksamkeitsnachweises [4] der «Spiraldynamik» erstaunen nicht. Die Benutzung der Spiralform der DNA-Helix und der Dynamik der Welle als wissenschaftliches Mäntelchen für die Prinzipien der «Spiraldynamik» kann nicht über die Absurdität von spiralförmigen Bewegungen als Therapiemethode hinwegtäuschen. Sie ist das Produkt von autistisch-undiszipliniertem Denken [3].

Bei dieser Sachlage ist es erstaunlich, dass es der Spiraldynamik Med Center AG gelang, in Basel, Bern und Zürich ein diagnostisch-therapeutisches Zentrum unter orthopädisch-fachärztlicher Leitung zu gründen [2]. Bei der selbstverständlichen Annahme, dass die Schulthessklinik und die Uniklinik Balgrist eine wissenschaftlich fundierte orthopädische Chirurgie betreiben, ist es unbegreiflich, dass diese Kliniken zu den regelmässigen Zuweisern von Patienten an das Spiraldynamik Med Center in Zürich gehören sollen [2]. Wenn diese Aussagen des medizinischen Leiters dieses Zentrums zutreffen, sind Zweifel an der ärztlichen Kompetenz der zuweisenden Ärzte berechtigt. Auch wenn die Physiotherapeutinnen, die mit den im Bereich des Bewegungsapparats tätigen Ärzten (orthopädische Chirurgen, Neurologen, Neurochirurgen, Rheumatologen, Internisten und Allgemeinpraktiker) zusammenarbeiten, in Abweichung zum früher Üblichen die Art und Weise des therapeutischen Vorgehens selbst bestimmen wollen, liegt eine enge Zusammenarbeit. zwischen kompetenten Ärzten und ebenso kompetenten Physiotherapeutinnen im Interesse der Patienten. Denn gutausgebildete und vernünftig denkende Physiotherapeutinnen respektieren die Grenzen des mit Physiotherapie und physikalischer Therapie Erreichbaren und distanzieren sich von schaumschlägerischen Behandlungspraktiken, die die Fakten der Anatomie, Funktionsweise und Pathologie des Bewegungsapparates nicht wahrhaben wollen.

Die vom «Fachleiter Qualität und Innovation Spiraldynamik» orthopädischen Chirurgen gemachten Vorwürfe, zu operationsfreudig zu sein [1], sind schon mehrfach $[4,8]$, aber nicht pauschal erhoben worden. Sie wirken aus der Feder eines Spiraldynamik-Anwenders tendenziös. Das spontane Verschwinden und die Remissionen von Beschwerden sind besonders im Bereiche des Rückens, der Knie und Füsse erfreulich häufig. Dies erklärt das Verschwinden von Beschwerden während der Wartezeit auf eine Arztkonsultation. Deswegen wären die als Folge des für 2020 prognostizierten Orthopädenmangels [6] entstehenden Wartelisten für Patienten, die unter nicht lebensbedrohenden Beschwerden leiden, gar nicht so bedenklich. Der Hinweis auf den gesetzlich verankerten Informed consent [5] ist für Patienten und alle Akteure im Gesundheitswesen nicht unangebracht. Aber es ist natürlich peinlich, dass es allzu therapiefreudige, operierende und nicht operierende Akteure im Gesundheitswesen gibt und dass diese bei Patienten, die das Zauberwort
«Therapie» fasziniert, nicht unbeliebt sind. Leider lassen sich leichtgläubige und verängstigte Patienten nicht immer beruhigen und auch durch eine verständnisvolle Information nicht vor dem Besuch eines Wunderheilers bewahren.

\section{Zusammenfassung}

Bei der sogenannten «Spiraldynamik» handelt es sich um ein Wissenschaftlichkeit vorspiegelndes, die DNA-Helix als Spirale und die Welle als dynamisches Prinzip verwendendes Behandlungskonstrukt, das die anerkannten anatomischen, physiologischen und pathologischen Fakten des Bewegungsapparates nicht beachtet, keinen Wirkungsnachweis vorlegen kann und die Selbstheilungserfolge des menschlichen Bewegungsapparates einem widernatürlichen spiralförmigen Bewegungsmuster zurechnet. Die SpiraldynamikÄrzte und -Therapeuten nützen die zeitgeistige Therapiegläubigkeit geschäftstüchtig aus. Leider hat die Mehrzahl der chirurgisch tätigen Orthopäden die nicht-operative Therapie und deren Überwachung aus der Hand gegeben. Anstelle der früheren erfolgreichen Zusammenarbeit mit seriös ausgebildeten, vernünftigen Physiotherapeutinnen lassen sie nicht operierende Orthopäden mit wirklichkeitsfremden Vorstellungen über den Bewegungsapparat geschäften und tragen damit zur weiteren Ausdehnung des bereits grossen Tummelfeldes für quacksalberische Praktiken im Bereiche des Bewegungsapparates bei $[7,8,9]$.

\section{Literatur}

1 Larsen C. Trainieren statt operieren? Schweiz Ärztezeitung. 2009;90(38):1476-99.

2 Larsen C. Zum Brief von Max Geiser: «Kritische Bemerkungen zum NFP53». Schweiz Ärztezeitung. 2011;92(6):195.

3 Bleuler E. Das autistisch-undisziplinierte Denken in der Medizin und seine Überwindung. Berlin: Springer; 1927.

4 Geiser M. Wie wissenschaftlich ist die Chirurgie? Schweiz Ärztezeitung. 1996;77:318-22.

5 Bucher E. Das Horror-Konstrukt der «Zwangsmedikation»: Zweimal (ohne Zuständigkeit) ein Ausflug ins juristische Nirwana (Zu BGE 126 I 6-30). Zeitschrift des Bernischen Juristenvereins. 2001;137(10):764-807.

6 Brandenberg JE. Orthopädenmangel 2020. Schweiz Ärztezeitung. 2011;92(18):676-8.

7 Geiser M. Der menschliche Körper - die letzte Bastion der Magie. Schweiz Ärztezeitung. 1988;69:1133-7.

8 Geiser M. Gedanken zur orthopädischen Chirurgie in der zweiten Hälfte des 20. Jahrhunderts. Schweiz Ärztezeitung. 2000;81(39):2233-8.

9 Skrabanek P, McCormick J. Follies and Fallacies in Medicine. Glasgow: The Terragon Press; 1989. 